\title{
Desenvolvimento motor grosso e as habilidades socioemocionais de bebês vulneráveis no primeiro trimestre de vida
}

\author{
Gross motor development and socioemotional skills of vulnerable babies in the first \\ trimester of life
}

Letícia Nadal da Silva ${ }^{1}$

Alexandra Quines Mendelski²

Carla Skilhan de Almeida ${ }^{3}$

Laís Rodrigues Gerzson ${ }^{4}$

Parecer de aprovação pelo comitê de ética: 3.381.618.

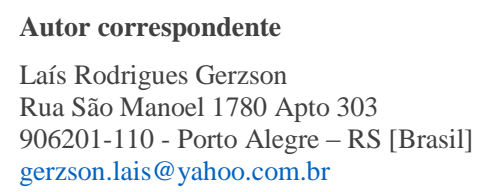

${ }^{1}$ Hospital Materno Infantil Presidente Vargas, Porto Alegre, RS, Brasil.

${ }^{2}$ Hospital Materno Infantil Presidente Vargas, Porto Alegre, RS, Brasil.

${ }^{3}$ Departamento de Fisioterapia, Educação Física e Dança, Universidade Federal do Rio Grande do Sul (UFRGS), Porto Alegre, RS, Brasil.

${ }^{4}$ Programa de Pós-Graduação Saúde da Criança e do Adolescente, Universidade Federal do Rio Grande do Sul (UFRGS), Porto Alegre, RS, Brasil.

\section{Cite como}

Vancouver

\section{Resumo}

Introdução: O desenvolvimento motor do bebê tem influências cognitivas, socioemocionais e ambientais.

Objetivo: verificar se existe associação dos escores do desenvolvimento motor grosso com as habilidades socioemocionais de bebês em seu primeiro trimestre de vida.

Método: Estudo transversal, composto por 20 bebês provenientes de um hospital público de Porto Alegre, RS. Para a avaliação dos fatores de risco sociodemográficos foi utilizado um questionário elaborado pelas pesquisadoras. A Bayley III foi utilizada para avaliar o desenvolvimento motor grosso e habilidades socioemocionais.

Resultados: Houve associação positiva estatisticamente significativa entre os escores brutos de motor grosso e socioemocional $(\mathrm{r}=0,665 ; \mathrm{p}=0,001)$, como também nos escores bruto de motor grosso com os escores composto e percentil da socioemocional $(r=0,571 ; p=0,008$ e $r=0,588 ; p=0,006$, respectivamente). Houve associação positiva estatisticamente significativa entre idade gestacional $(\mathrm{p}=0,028)$, idade do bebê $(\mathrm{p}=0,003)$ e peso ao nascer $(\mathrm{p}=0,024)$ com o escore bruto motor grosso.

Conclusão: Houve associação significativa positiva nos escores do desenvolvimento motor grosso e habilidades socioemocionais.

Palavras-chave: Desenvolvimento infantil. Fisioterapia. Saúde da criança.

\begin{abstract}
Introduction: Babies' motor development has cognitive, socioemotional, and environmental influences.

Objective: To verify if there is an association between gross motor development scores and socioemotional skills of babies in their first trimester of life.

Method: Cross-sectional study done with 20 babies from a public hospital in the city of Porto Alegre, RS. In order to assess sociodemographic risk factors, a questionnaire prepared by the researchers was used. Bayley-III was used to assess gross motor development and socioemotional skills.

Results: There was a statistically significant positive association between gross motor development and socioemotional skills scores $(r=0.665 ; p=0.001)$, as well as in gross motor development scores with socioemotional composite and percentile scores $(r=0.571 ; p=0.008$ and $r=0.588 ; p=0.006$, respectively). There was a statistically significant positive association between gestational age $(\mathrm{p}=0.028)$, baby age $(\mathrm{p}=0.003)$, and birth weight $(\mathrm{p}=0.024)$ with the gross motor development score.

Conclusion: There was a significant positive association between gross motor development and socioemotional skills scores.
\end{abstract}

Keywords: Child development. Physical Therapy Specialty. Child health.

Silva, LN, Mendelski, AQ, Almeida, CS, Gerzson, LR. Desenvolvimento motor grosso e as habilidades socioemocionais de bebês vulneráveis no primeiro trimestre de vida. Conscientiae Saúde 2019 out./dez.;18(4):489-506.

https://doi.org/10.5585/conssaude.v18n4.15900. 


\section{Introdução}

O desenvolvimento infantil é um processo que se inicia na vida intrauterina, o qual envolve o crescimento físico, a maturação neurológica e as aquisições de habilidades relacionadas ao comportamento do bebê $\hat{e}^{1}$. Os primeiros anos de vida caracterizam-se como uma etapa de suma importância no desenvolvimento global do ser humano, devido a sua "tecelagem neuronal", que acontece em grande velocidade, bilhões de sinapses vão expandindo as percepções do recém-nascido e formando sua visão de si mesmo, dos outros e do contexto em que habita ${ }^{2}$.

Profissionais que atuam com esse desenvolvimento têm uma ferramenta muito importante para avaliar o desenvolvimento global do nuelo: as suas habilidades motoras no início da vida. Essas habilidades expressa a integridade do sistema nervoso e a influência dos estímulos externos, sejam eles sociais, cognitivos ou emocionais ${ }^{3}$. Diferentes fatores podem exercer influência sobre o desenvolvimento motor, como os fatores socioeconômicos, biológicos e maternos ${ }^{4,5,6}$. O reconhecimento disso nas interações entre o neonato, a família e o contexto amplia a compreensão da necessidade de uma abordagem multidimensional no estudo do desenvolvimento humano ${ }^{7,8}$.

O desenvolvimento motor do bebê pode ser dividido em trimestres, o qual cada trimestre apresenta características específicas. Se esse bebê está saudável, ele desenvolverá a capacidade de levantar e girar a cabeça de um lado para o outro em uma fase mais inicial ${ }^{9}$. No primeiro mês de vida, apresenta membros flexionados, cabeça oscilante e mãos fechadas em supino, além disso, o tronco mais hipotônico e presença de movimentos amplos, fluentes, complexos e variáveis (General Movements -GMs) com influência de reflexos primitivos. No segundo mês, pode apresentar uma postura mais assimétrica; em decúbito ventral, eleva mais a cabeça, mas não a mantém erguida, e os membros inferiores estarão um pouco mais estendidos. No final do terceiro mês, espera-se aquisição do equilíbrio cervical; consegue manter a cabeça na linha média e realizar rotação da cabeça para ambos os lados. Há, ainda, um aumento de movimento com menos intensidade, força e velocidade, com começo e fim gradual (Fidgety Movements) e, Em prono, consegue fazer descarga de peso em antebraços, elevando o tronco e a cabeça na linha média ${ }^{10,11}$.

Quanto às habilidades socioemocionais, durante a primeira infância, são descritas como a capacidade emergente da criança de experimentar, controlar e expressar sentimentos; formar conexões interpessoais próximas e seguras; investigar o meio ambiente e aprender com ele. $\mathrm{Na}$ medida em que os nuelos desenvolvem-se, as expressões emocionais tornam-se mais 
organizadas, em parte, devido ao apoio dos cuidadores. Ao longo dos primeiros anos de vida, esses tornam-se progressivamente capazes de entender as próprias emoções e as de outros sujeitos, participando de uma comunicação intencional e compartilhando-a deliberadamente com outras pessoas. O desenvolvimento socioemocional do bebê depende da acessibilidade de um ambiente saudável; uma alimentação adequada; uma cooperação amável e ponderada por meio de figuras parentais; e de oportunidades de aprendizado ${ }^{12}$.

Os fatores ambientais associados ao desenvolvimento motor têm se destacado fortemente na literatura, sugerindo que o ambiente é capaz de modular os riscos a que os recémnascidos estão expostos. Os ambientes positivos são aqueles que desafiam os neonatos a se desenvolverem nas suas aquisições motoras ${ }^{13}$. Em contrapartida, ambientes vulneráveis podem modificar o rumo do desenvolvimento. Considera-se um ambiente vulnerável aquele que apresenta situações socioeconômicas como pobreza, abuso e violência; baixa escolaridade dos pais; condições precárias de moradia; desnutrição; e falta de acesso a recursos educacionais e saudáveis ${ }^{14}$.

O profissional, muitas vezes, diante da ausência de experiências e oportunidades ofertadas ao bebê, precisa suprir essa situação por meio da avaliação e da intervenção precoce, a fim de minimizar os futuros prejuízos na vida desse bebê ${ }^{15,16}$. Assim, quando deparar-se com um ambiente hospitalar em que o público que foi atendido é o bebê extremamente vulnerável, preocupa-se com seus aspectos de desenvolvimento global. A questão foi formulada no fato de: como estão os aspectos motores grossos desses bebês vulneráveis? Como estão os aspectos socioemocionais dos bebês que foram acompanhados? Será que existe uma associação entre esses dados? Diante dessas questões, o objetivo principal deste estudo, além de analisar os aspectos socioemocionais e os aspectos motores grossos, é verificar se existe associação dos escores do desenvolvimento motor grosso com as habilidades socioemocionais de bebês em seu primeiro trimestre de vida.

\section{Metodologia}

\section{Delineamento e participantes}

Este artigo se caracteriza como um estudo de tipo transversal. O cálculo do tamanho da amostra foi realizado pelo software WinPEPI versão 11.43. Para um nível de significância de $5 \%$, um poder de $90 \%$ e um coeficiente de correlação mínimo de 0,7 entre as escalas obteve-se um total mínimo de 17 bebês. A coleta de dados foi realizada entre os meses de junho a setembro de 2019, sendo a amostra constituída pelos pacientes do ambulatório de fisioterapia do Hospital 
Materno Infantil Presidente Vargas (HMIPV), em Porto Alegre-RS, que haviam tido alta hospitalar e permaneciam em acompanhamento. Os critérios de inclusão estabelecidos foram: (a) ter de um a três meses de vida; (b) estar em acompanhamento no ambulatório de fisioterapia do HMIPV; e (c) retornar o termo de consentimento informado assinado pelos responsáveis legais do bebê, antes do início da avaliação. Todos os bebês que nasceram prematuros tiveram correção da idade no momento da avaliação motora. Foram excluídos da pesquisa: (a) bebês com doenças genéticas, sindrômicas, neurológicas; (b) disfunções respiratórias graves; ou (c) que, por algum motivo, não atenderam aos critérios de inclusão.

\section{Procedimentos e instrumentos de avaliação}

Inicialmente, para obtenção das informações gerais sobre o bebê, utilizou-se um questionário de identificação e dados demográficos, elaborado pelas pesquisadoras, com base na literatura atual ${ }^{14,17}$, que aborda de forma ampla os fatores sociais, emocionais, biológicos e ambientais, os quais podem ser considerados fatores de risco ao desenvolvimento motor. O interrogatório apresenta as principais informações de saúde do bebê, desde a gestação até o presente acompanhamento no ambulatório, bem como, informações relacionadas à saúde dos familiares e dados demográficos. Ele teve duração aproximada de 20 minutos e foi realizado na forma de entrevista. As avaliações foram conduzidas em uma sala do setor, específica para esse fim, com mobílias que permitissem a movimentação espontânea e segura dos bebês.

Em seguida, para a avaliação do desenvolvimento motor grosso e das habilidades socioemocionais foi utilizada a Bayley Scales of Infants and Oder Development - 3rd edition (Bayley III), que é um instrumento de observação traduzido, adaptado e validado para a população brasileira ${ }^{18}$. A Bayley III é composta por 602 itens distribuídos em cinco dimensões do desenvolvimento infantil: a) escala cognitiva; b) escala de linguagem (linguagem receptiva e expressiva); c) escala motora (motor grosso e fino); d) escala socioemocional; e e) comportamento adaptativo. Os três primeiros são avaliados através da observação direta do bebê em situações de teste, enquanto os dois últimos são avaliados através de questionários a serem preenchidos pelo cuidador principal. A escala não fornece uma pontuação total geral, mas separa as pontuações brutas e escaladas para cada domínio, bem como, as pontuações compostas e as classificações percentuais para cada escala.

A Bayley III pontua o desempenho da criança de acordo com sua idade, e para cada idade há um ponto de início dos itens a serem administrados. O escore bruto é dado pela soma de todos os itens para os quais o bebê recebeu crédito, acrescido da soma dos itens das idades 
anteriores. Através do escore bruto, é possível se obter o escore escalonado e o escore composto (escores padronizados). A classificação do desempenho da criança é feita através do escore composto: muito superior (> 130), superior (120-129), médio alto (110-119), médio (90-109), médio baixo (80-89), limítrofe (70-79) e extremamente baixo $(<69)$.

Importante compreender que, em cada dimensão, a escala separa por letras (de A ao Q) que são referentes a idades aproximadas e, cada item, soma-se um se a criança realizou ou zero se não realizou as atividades propostas. Com um foco intencional, no presente estudo, foi utilizado apenas a escala que avalia o domínio motor grosso e as habilidades socioemocionais. A escala motora grossa é composta por 72 itens que avalia o movimento dos membros e do tronco, o posicionamento estático (sentar, e levantar), os movimentos dinâmicos, incluindo locomoção e coordenação, balance (equilíbrio) e planejamento motor. No primeiro trimestre de vida, a escala motora grossa (zero a três meses) considera as letras de $\mathrm{A}$ a $\mathrm{D}$, em que a pontuação vai de zero a $14^{18}$.

A escala socioemocional avalia a aquisição de marcos de desenvolvimentos sociais e emocionais em bebês e crianças pequenas, identificando os principais marcos que devem ser alcançados até certas idades, tornando possível, também, verificar no primeiro trimestre de vida: "a facilidade de estabelecer vínculos e desenvolver confiança; a diversidade de tipos de choros; a fixação do olhar; a procura; a reação dos estímulos auditivos e visuais; a vocalização; o sorriso; e a demonstração de frustação e raiva" ${ }^{19}$. As perguntas realizadas com os pais avaliam o domínio da criança com relação a habilidades emocionais- funcionais, tais como: a autorregulação e o interesse no mundo; a comunicação de necessidades; a interação com outros e o estabelecimento de relações, utilizando as emoções como forma interativa e proposital; e o uso de sinais emocionais ou de gestos para resolver problemas. Os itens de um a oito dessa escala avaliam as capacidades de processamento sensorial da criança (sensibilidade a cores, sons, toque ou movimentos $)^{17}$. Em relação à escala socioemocional, no primeiro trimestre de vida (zero a três meses), a pontuação socioemocional bruta vai de zero a 55 , a pontuação composta vai de 55 a 135 , com um percentil de 0,1 a $99^{18}$.

Os avaliadores foram cegados e houve treinamento prévio de duas semanas com doutores na área. O estudo foi submetido e aprovado pelo Comitê de Ética em Pesquisa do Hospital Materno Infantil Presidente Vargas (CEP-HMIPV), com o número do parecer: 3.381.618 (CAEE 13139219.0.0000.5329). 


\section{Análise estatística}

A descrição dos dados foi realizada por meio de frequências absolutas (n) e relativas (\%), para variáveis qualitativas, e por média e desvio padrão para variáveis quantitativas, em caso de distribuição simétrica. Caso ocorra distribuição assimétrica ela é adequada à utilização da mediana e da amplitude interquartílica. Aplicou-se o teste de Shapiro - Wilk para verificar a normalidade dos dados. Para avaliar a associação entre as escalas motora de Bayley III, socioemocional e dados demográficos, os testes da correlação de Pearson ou Spearman foram aplicados. Também, foram utilizados os testes $t$-student e Análise de Variância (ANOVA) para comparar médias. Para todas as análises foi utilizado o software Statistical Package for the Social Sciences (SPSS) versão 22.0, considerando um nível de significância de 5\%.

\section{Resultados}

Foram avaliados 20 bebês em acompanhamento no ambulatório, com média de idade de 2,5 meses ( $\mathrm{DP} \pm 0,7)$, sendo 11 meninas $(55,0 \%)$. Quanto à idade gestacional, oito eram prematuros $(40,0 \%)$, com idade inferior a 37 semanas. Dos bebês avaliados, quatro $(20,0 \%)$ deles eram gemelares, e 16 bebês $(80,0 \%)$ tinham amamentação exclusiva. Ainda, notou-se que os bebês do ambulatório haviam internado por problemas respiratórios, em sua maioria $(\mathrm{n}=18)$ por bronquiolite $(94,7 \%)$, sendo que oito $(40,0 \%)$ já apresentavam internações anteriores (Tabela 1). 
Tabela 1 - Caracterização da amostra.

\begin{tabular}{|c|c|}
\hline Variáveis & $\mathrm{n}=\mathbf{2 0}$ \\
\hline Idade Gestacional (sem) - média \pm DP & $36,9 \pm 3,1$ \\
\hline Idade do bebê (meses) - média \pm DP & $2,5 \pm 0,7$ \\
\hline Peso ao nascer $(\mathrm{g})-$ média $\pm \mathrm{DP}$ & $2852 \pm 743$ \\
\hline Altura ao nascer $(\mathrm{cm})-$ média $\pm \mathrm{DP}$ & $46,5 \pm 4,0$ \\
\hline Peso atual $(\mathrm{g})-$ média $\pm \mathrm{DP}$ & $5351 \pm 940$ \\
\hline Altura atual $(\mathrm{cm})-$ média \pm DP & $57,5 \pm 4,2$ \\
\hline Prematuridade $-\mathrm{n}(\%)$ & $8(40,0)$ \\
\hline Gemelar - n $(\%)$ & $4(20,0)$ \\
\hline \multicolumn{2}{|l|}{ Sexo $-\mathrm{n}(\%)$} \\
\hline Masculino & $9(45,0)$ \\
\hline Feminino & $11(55,0)$ \\
\hline \multicolumn{2}{|l|}{ Raça do bebê - n(\%) } \\
\hline Branca & $15(75,0)$ \\
\hline Parda & $4(20,0)$ \\
\hline Preta & $1(5,0)$ \\
\hline APGAR $1^{\circ}$ minuto - mediana $(\mathrm{P} 25-\mathrm{P} 75)$ & $8(8-8,5)$ \\
\hline APGAR $5^{\circ}$ minuto - mediana (P25-P75) & $9(9-9)$ \\
\hline AM Exclusivo - n $(\%)$ & $16(80,0)$ \\
\hline \multicolumn{2}{|l|}{ Motivo da internação $(\mathrm{n}=19)-\mathrm{n}(\%)$} \\
\hline Hemorragia pulmonar & $1(5,3)$ \\
\hline Bronquiolite & $18(94,7)$ \\
\hline Internações hospitalares anteriores $-\mathrm{n}(\%)$ & $8(40,0)$ \\
\hline
\end{tabular}

Legenda: sem: semanas; g: gramas, cm: centímetros; DP: desvio padrão, n: número, AM: amamentação Fonte: Elaborado pelas autoras.

Quanto às características dos pais, os resultados estão demonstrados na Tabela 2. Todas as moradias possuem cômodos e o principal cuidador da criança é a mãe. Entre as profissões maternas, a mais prevalente foi do lar/dona de casa (55\%), e, entre as profissões paternas, as mais prevalentes foram, respectivamente, serviços gerais $(15,8 \%)$, segurança $(10,5 \%)$, pedreiro $(10,5 \%)$ e desempregados (10,5\%). Entre as atividades dos bebês, 15 (75\%) ficam deitados em decúbito dorsal pela manhã, três $(15 \%)$ sentados e dois (10\%) ficam brincando. Pela tarde, 10 (50\%) ficam deitados, três (15\%) sentados, um (5,0\%) em pé e seis (30\%) brincando. Pela noite, todos (100\%) estão deitados. 
Tabela 2 - Caracterização dos pais

\begin{tabular}{|c|c|}
\hline Variáveis & $\mathrm{n}=\mathbf{2 0}$ \\
\hline Idade materna (anos) - média \pm DP & $23,8 \pm 5,0$ \\
\hline Idade paterna (anos) - média \pm DP & $28,7 \pm 6,9$ \\
\hline \multicolumn{2}{|l|}{ Raça materna - n $(\%)$} \\
\hline Branca & $12(60,0)$ \\
\hline Parda & $3(15,0)$ \\
\hline Preta & $5(25,0)$ \\
\hline \multicolumn{2}{|l|}{ Raça paterna $(n=19)-n(\%)$} \\
\hline Branca & $12(63,2)$ \\
\hline Parda & $3(15,8)$ \\
\hline Preta & $4(21,1)$ \\
\hline Escolaridade materna (anos) - média \pm DP & $9,6 \pm 2,2$ \\
\hline Escolaridade paterna (anos) - média \pm DP & $8,7 \pm 3,1$ \\
\hline \multicolumn{2}{|l|}{ Estado civil materno $-\mathrm{n}(\%)$} \\
\hline Solteira & $12(60,0)$ \\
\hline Casada/união estável & $7(35,0)$ \\
\hline Divorciada & $1(5,0)$ \\
\hline Número de pessoas que moram na casa - média \pm DP & $5,6 \pm 1,5$ \\
\hline Pais moram juntos $-\mathrm{n}(\%)$ & $15(75,0)$ \\
\hline Cuidador trabalha fora $-\mathrm{n}(\%)$ & $5(25,0)$ \\
\hline Renda familiar (reais) - mediana (P25-P75) & $1200(900-2200)$ \\
\hline \multicolumn{2}{|l|}{ Moradia $-\mathrm{n}(\%)$} \\
\hline Própria & $14(70,0)$ \\
\hline Alugada & $4(20,0)$ \\
\hline Cedida & $2(10,0)$ \\
\hline \multicolumn{2}{|l|}{ Material - n(\%) } \\
\hline Alvenaria & $16(80,0)$ \\
\hline Madeira & $4(20,0)$ \\
\hline Moradia possui recursos básicos $-\mathrm{n}(\%)$ & $18(90,0)$ \\
\hline \multicolumn{2}{|l|}{ Dormitório $-\mathrm{n}(\%)$} \\
\hline Berço & $7(35,0)$ \\
\hline Cama dos pais & $11(55,0)$ \\
\hline Outro & $2(10,0)$ \\
\hline \multicolumn{2}{|l|}{ Parto - n (\%) } \\
\hline Cesáreo & $8(40,0)$ \\
\hline Vaginal & $12(60,0)$ \\
\hline
\end{tabular}

Legenda: DP: desvio padrão, n: número

Fonte: Elaborado pelas autoras.

De acordo com a escala Bayley III, foi possível identificar que os bebês estão dentro da normalidade na escala motora grossa na pontuação bruta, como também, na escala socioemocional nas pontuações bruta, composta e percentílica (Tabela 3). 
Tabela 3 - Dados sobre a Bayley III

\begin{tabular}{lc}
\hline Variáveis & $\mathbf{n = 2 0}$ \\
\hline Motor Grosso - média \pm DP & $13,4 \pm 4,1$ \\
$\quad$ Pontuação bruta & \\
Escala Socioemocional - média \pm DP & $48,5 \pm 4,2$ \\
$\quad$ Pontuação bruta & $111,8 \pm 8,3$ \\
Pontuação composta & $75,4 \pm 16,0$ \\
Percentil
\end{tabular}

Legenda: DP: desvio padrão

Fonte: Elaborado pelas autoras.

Houve associação positiva estatisticamente significativa entre os escores brutos de motor grosso e de socioemocional $(r=0,665 ; \mathrm{p}=0,001)$, conforme apresenta a Figura 1, ou seja, quanto maior o escore total bruto, maior a pontuação socioemocional. A mesma associação positiva significativa foi encontrada entre os escores bruto de motor grosso com os escores composto e percentil da socioemocional ( $\mathrm{r}=0,571 ; \mathrm{p}=0,008$ e $\mathrm{r}=0,588 ; \mathrm{p}=0,006$, respectivamente).

Figura 1 - Associação entre as pontuações brutas totais dos escores de motor grosso e socioemocional

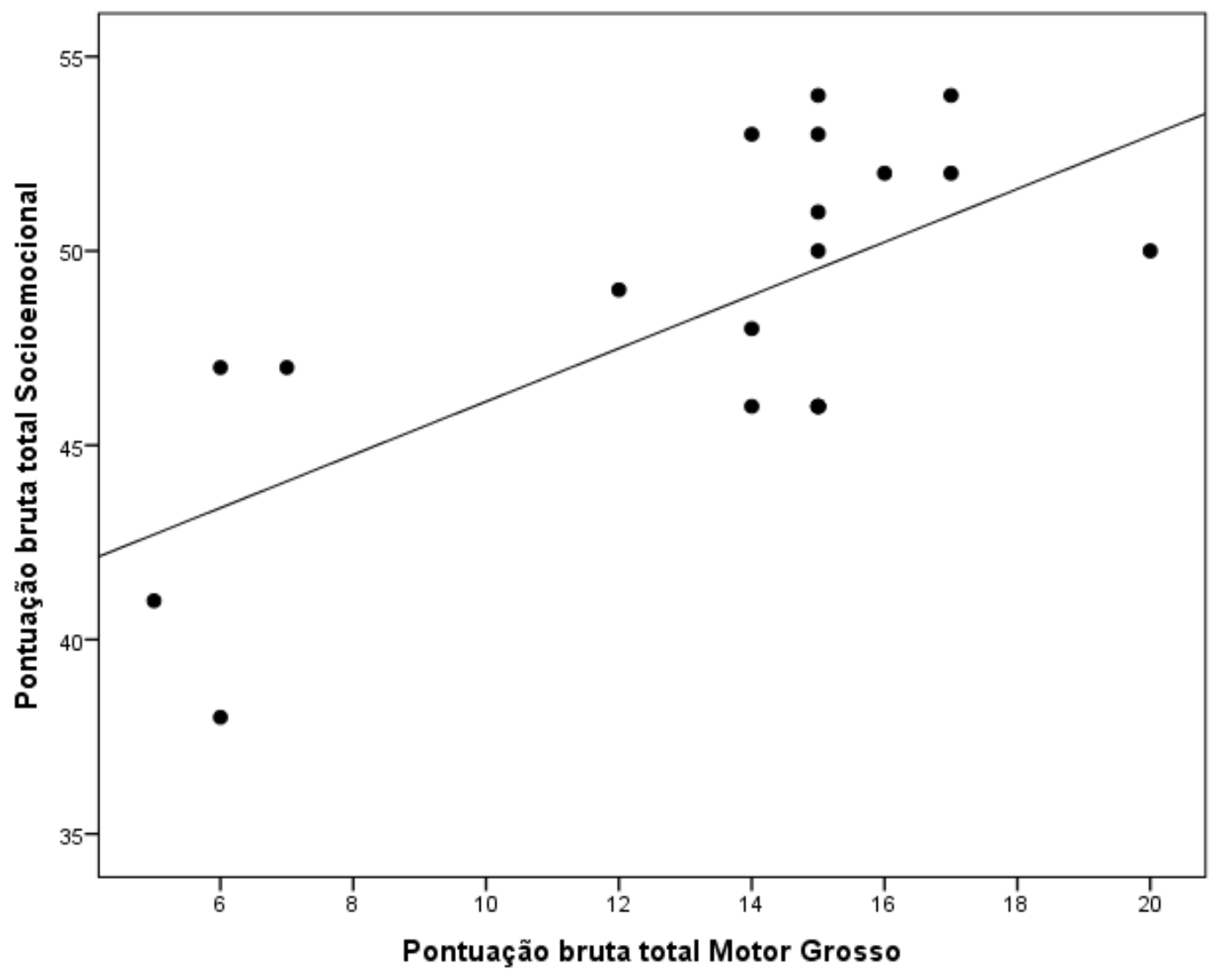

Fonte: Elaborado pelas autoras. 
Conforme apresenta a Tabela 4 abaixo, houve associação positiva estatisticamente significativa entre idade gestacional $(\mathrm{p}=0,028)$, idade do bebê $(\mathrm{p}=0,003)$ e peso ao nascer ( $\mathrm{p}=0,024)$, com o escore bruto motor grosso, ou seja, quanto maior a idade gestacional, a idade do bebê e o peso ao nascer, maior o escore bruto da escala motor grosso.

Testes de associação foram realizados para a prematuridade, a amamentação exclusiva, a gemelaridade, os pais que moram juntos, o cuidador que trabalha fora, a moradia em que o bebê reside, o dormitório, o sexo do bebê e o tipo de parto; e essas associações não foram estatisticamente significativas $(\mathrm{p}>0,05)$.

Tabela 4 - Associações entre os escores da Bayley III com dados sociodemográficos.

\begin{tabular}{lcc}
\hline Variáveis & $\begin{array}{c}\text { Escore bruto motor } \\
\text { grosso }\end{array}$ & $\begin{array}{c}\text { Escore percentil } \\
\text { socioemocional }\end{array}$ \\
\hline Idade Gestacional & $\mathbf{r}=\mathbf{0 , 4 9 0}(\mathrm{p}=\mathbf{0 , 0 2 8})$ & $\mathrm{r}=0,316(\mathrm{p}=0,175)$ \\
Idade do bebê (meses) & $\mathrm{r}=\mathbf{0 , 6 3 1}(\mathrm{p}=\mathbf{0 , 0 0 3})$ & $\mathrm{r}=0,244(\mathrm{p}=0,300)$ \\
Peso ao nascer (g) & $\mathrm{r}=\mathbf{0 , 5 0 3}(\mathrm{p}=\mathbf{0 , 0 2 4})$ & $\mathrm{r}=0,348(\mathrm{p}=0,133)$ \\
APGAR 1 ${ }^{\circ}$ minuto & $\mathrm{r}_{\mathrm{s}}=0,109(\mathrm{p}=0,676)$ & $\mathrm{r}_{\mathrm{s}}=0,235(\mathrm{p}=0,364)$ \\
APGAR 5 ${ }^{\circ}$ minuto & $\mathrm{r}_{\mathrm{s}}=0,414(\mathrm{p}=0,099)$ & $\mathrm{r}_{\mathrm{s}}=0,318(\mathrm{p}=0,214)$ \\
Idade materna (anos) & $\mathrm{r}=0,284(\mathrm{p}=0,225)$ & $\mathrm{r}=0,259(\mathrm{p}=0,270)$ \\
Idade paterna (anos) & $\mathrm{r}=0,119(\mathrm{p}=0,637)$ & $\mathrm{r}=0,135(\mathrm{p}=0,594)$ \\
Escolaridade materna (anos) & $\mathrm{r}=-0,204(\mathrm{p}=0,389)$ & $\mathrm{r}=-0,241(\mathrm{p}=0,306)$ \\
Escolaridade paterna (anos) & $\mathrm{r}=0,066(\mathrm{p}=0,802)$ & $\mathrm{r}=-0,236(\mathrm{p}=0,362)$ \\
Número de pessoas que moram na casa & $\mathrm{r}=0,180(\mathrm{p}=0,448)$ & $\mathrm{r}=0,363(\mathrm{p}=0,116)$ \\
Renda familiar (reais) & $\mathrm{r}=-0,228(\mathrm{p}=0,349)$ & $\mathrm{r}=-0,153(\mathrm{p}=0,533)$
\end{tabular}

Legenda: g: gramas

Fonte: Elaborado pelas autoras.

\section{Discussão}

No início da vida de um bebê, a avaliação do desenvolvimento global, principalmente, o motor, pode ser uma ferramenta precisa para verificar se ele bebê está íntegro ou não. Os bebês recém-nascidos já são capazes de interpretar informações sensoriais e usá-las para modificar movimentos e organizar o sistema de controle postural, baseado nas demandas da $\operatorname{tarefa}^{20,21}$. Assim, a maior preocupação foi verificar precocemente como estava o desenvolvimento dessa população tão vulnerável, bem como, suas questões socioemocionais, e a existência de uma possível associação entre essas variáveis. 
Como citado anteriormente, a pobreza, o abuso e a violência; a baixa escolaridade dos pais; as condições precárias de moradia; a mãe solo; a desnutrição; o parto prematuro, o baixo peso ao nascimento e a falta do aleitamento materno exclusivo; as internações hospitalares; e a escassez de recursos em educação e de saúde são condições de vulnerabilidades ${ }^{13}$. Os bebês deste estudo apresentaram muitos dos requisitos de vulnerabilidade.

Alguns autores acreditam que o repertório motor do bebê, nos primeiros dois meses após a idade de termo, é uma continuação do comportamento fetal, e novas funções são adicionadas a esse repertório, como, por exemplo, respostas vestibulares e visuais, reações de retificação e refinamento de movimentos gerais. Movimentos de pequena à moderada amplitude, de velocidade lenta à moderada, com sequência variável de movimentos do braço, da perna, do pescoço e do tronco dão lugar a um aumento de movimento com menos intensidade, força e velocidade, com começo e fim gradual. Por volta de nove semanas de idade pós-termo, época da grande transformação, um novo tipo de movimentos gerais aparece: os fidgety movements ${ }^{10,20,22}$, que podem ser vistos das nove semanas pós-nascimento até os quatro meses, momento em que iniciam os movimentos voluntários e antigravitacionais. O desenvolvimento motor típico é representado por períodos de ajuste e variabilidade das atividades motoras realizadas pelo bebêt $\hat{e}^{10,23}$.

No Brasil, vem crescendo os estudos que descrevem a trajetória motora de bebês até quatro meses de idade; entretanto, ainda existe um pobre diagnóstico precoce de alterações no desenvolvimento motor nessa faixa etária. Acredita-se que muitos outros estudos devam ser realizados com bebês de risco. Por sentir essa necessidade, a escala de Bayley III foi utilizada neste estudo com o intuito de verificar o desenvolvimento motor grosso dessa população. A média do escore bruto da avaliação motora grossa dos bebês avaliados estavam condizententes com os bebês da sua faixa etária, mesmo com todos os aspectos de vulnerabilidade. Sabe-se que esses bebês devem continuar sendo acompanhados pela equipe de saúde de sua comunidade.

Outros autores relatam que, no primeiro ano de vida, aspectos ambientais influenciam o desenvolvimento do bebê, como a idade e as práticas maternas, o grau de instrução dos pais, a renda familiar, as condições de moradia e as oportunidades ambientais para o desenvolvimento. Esses aspectos podem influenciar nas habilidades socioemocionais do beb $\hat{e}^{20}$. Essas habilidades se relacionam com a relação-dependência do vínculo estabelecido entre os bebês e seus cuidadores. Neste estudo, assim como nos aspectos motores desses bebês vulneráveis, as médias do escore bruto, composto e do percentil dos bebês avaliados quanto às habilidades socioemocionais estavam condizententes com os bebês da sua idade. 
Tendo como premissa que tanto os aspectos motores grosso quanto as habilidades socioemocionais, os recém-nascidos estavam semelhantes às características dos bebês típicos de sua idade, realizou-se a associação entre essas duas variáveis. Como pôde ser observado nos gráficos, os bebês obtiveram associação positiva quanto a desenvolvimento motor grosso e habilidades socioemocionais, isto é, quanto maior a pontuação do escore bruto motor grosso, maior as habilidades socioemocionais. Também obtiveram associação, com resultados significativos, a idade gestacional (quanto maior a idade gestacional ao nascimento, melhor os escores), a idade do bebê e o peso ao nascer quando comparados com o motor grosso. Caso o desenvolvimento motor grosso e as habilidades socioemocionais obtivessem alterações, ou ainda, se a associação entre eles fosse negativa, teria-se efetivamente um grupo de risco estabelecido.

$\mathrm{Na}$ pesquisa, foram realizados testes de associação para determinados tópicos de vulnerabilidade como: sexo do bebê, tipo de parto, aleitamento exclusivo, gravidez gemelar, pais residirem juntos, cuidador principal estar empregado, cômodos e residência, porém, as associações não foram estatisticamente significantes.

A idade gestacional associou-se positivamente com o escore bruto motor grosso. Quanto maior a idade gestacional, melhor a pontuação motora. Em estudos, como de Ribeiro et al. ${ }^{24}$, evidenciou-se que baixo peso e idade gestacional estão associados a sequelas motoras e atraso no desenvolvimento, isto porque o período intrauterino é crítico para o crescimento, e a prematuridade oferece possibilidade de interferência nos processos maturacionais cerebrais, levando a alterações anatômicas e estruturais, as quais acarretam déficits funcionais. Além disso, os bebês prematuros apresentam pouca variabilidade de movimento e controle postural diminuído em comparação aos bebês a termo ${ }^{19}$, e essa diminuição de movimentos limita a adaptação desses bebês às mudanças nas demandas das tarefas, resultando em atraso de aquisições motoras. Essas aquisições, em bebês a termo e pré-termo, seguem trajetórias diferentes ${ }^{25}$.

O peso ao nascimento, também, associou-se positivamente com o escore bruto motor grosso. Estes dois fatores, idade gestacional e peso baixo, podem levar o bebê a reinternações. Um percentil de $40 \%$ dos bebês já havia sido internado anteriormente, levando em conta que eles estavam no primeiro trimestre de vida, considera-se um número elevado. Silva et al. ${ }^{26}$ observaram que a prematuridade e o baixo peso podem estar associados a maior número de reinternações hospitalares no primeiro ano de vida, levando a prejuízos nas aquisições de habilidades do indivíduo devido a longos períodos de internação em ambientes vulneráveis. 
As famílias estudadas aqui evidenciaram a vulnerabilidade social e econômica através da renda familiar baixa e nível educacional baixo. O nível educacional é um fator de risco para alterações no desenvolvimento por estar associado ao cuidado oferecido ao bebê $\hat{e}^{6}$. Cuidadores mais esclarecidos estabelecem melhor vínculo, cuidado e possibilidade de estímulos. Além disso, a escolaridade está diretamente interligada com melhores colocações no mercado de trabalho, consequentemente, maior renda financeira. Baixos níveis educacionais associam-se com baixa renda e maior risco no desenvolvimento infantil.

Porém, acredita-se que essas famílias tinham características protetoras, o que auxiliou nos resultados positivos no desenvolvimento motor grosso e habilidades socioemocionais. A forma de cuidar dos bebês envolvidos era individual e estruturada, influenciando diretamente nos vínculos pré-estabelecidos em seu ambiente familiar. Apresentavam boas condições de moradia, a maioria dos bebês moravam com os pais, a mãe ficava tempo integral com seu bebê, e se consideravam com acesso à saúde, ao lazer e à cultura, corroborando com estudo prévio ${ }^{14}$.

O Brasil encontra-se em situação de subdesenvolvimento que evidencía diferenças sociais, econômicas e culturais diretamente relacionadas com o desenvolvimento infantil. A precaridade de estímulos e de incentivos no processo de maturação leva a recorrentes e significativos distúrbios a esse mecanismo próprio da maturação. O sujeito/cuidador é um ser social, influenciado pelo meio que vive ${ }^{27}$, por isso, é de suma importância vigiar esse processo.

Outro aspecto de proteção verificado neste estudo foi que $80 \%$ dos neonatos realizavam a amamentação exclusiva. $\mathrm{O}$ aporte de nutrientes é fundamental nos primeiros meses de vida, modulando processos associados à maturação da estrutura e da atividade cerebral, interferindo na trajetória do desenvolvimento infantil. Sabe-se que o aleitamento materno exclusivo pode ser considerado fator de proteção para desenvolvimento e desempenho motor, condição essa que fortalece a autoconfiança e traz satisfação à mulher. Esse ato faz aflorar sensações prazerosas de afetividade, e as mães que amamentam parecem mais responsivas aos sinais do filho durante os processos de interação no início da vida ${ }^{13}$. Em relação a bebês que utilizam fórmula infantil, associa-se índices medianos nos escores socioemocionais, isso porque, ao amamentar, estabelece-se o vínculo mãe-bebê e o contato físico entre ambos, o que estimula diretamente as habilidades do indivíduo ${ }^{12}$.

No que se refere às questões de moradia, os bebês do estudo obtiveram outros aspectos positivos, em que $75 \%$ moravam com várias pessoas na casa, os pais moravam juntos, a moradia era própria com saneamento básico e a casa era casa de alvenaria. O estímulo familiar é preditor para o desenvolvimento infantil, pesquisadores apontam que famílias numerosas facilitam o desenvolvimento do bebê principalmente no âmbito de habilidades motoras, isso deve-se à 
interação do bebê com uma proporção maior de diferentes estímulos ${ }^{28}$. Em estudo prévio, existe uma incidência de atrasos em bebês com famílias desfavorecidas socioeconomicamente, com menor espaço físico, pouca oferta de brinquedos e tempo de interação com os bebês/cuidador diminuídos ${ }^{13}$.

Detectou-se, ainda, neste estudo, que 55\% dos bebês dormiam junto com os pais, dividindo a mesma cama ("cama compartilhada" ou " co-leito"), provavelmente por sentiremse mais confortáveis com a situação e poderem cuidar seu filho no período noturno. Esse fato está relacionado com aspectos climáticos, valores culturais (síndrome da morte súbita), econômicos (tamanho da família, disponibilidade de espaço no domicilio) e alimentares (prevalência do aleitamento materno e sua manutenção) em cada família. No Brasil, a prática é comum em grupos de vulnerabilidade; porém, o estudo, não foi possível verificar essa associação com o desenvolvimento motor grosso, como também, nos aspectos socioemocionais. A literatura afirma que as crianças pequenas, que dormem com os pais, tendem a ser menos ansiosas, terem maior autoestima, tornando-se mais sociáveis, o que causa positivo impacto no desenvolvimento cerebral do bebê, e tendem a apresentar melhor comportamento escolar. Apesar disso, há restrições negativas para a independência da criança, já que não proporciona a prática de dormir sozinha, além de poder interferir negativamente no relacionamento matrimonial dos pais ${ }^{29}$.

O Fundo das Nações Unidas para a Infância (UNICEF) recomenda que o bebê compartilhe o mesmo quarto com os pais, pelo menos até os primeiros seis meses de vida, pois o local mais seguro para o bebê dormir, nesse período, é em um berço ao lado da cama. Colocar o berço ao lado da cama dos pais proporciona um ambiente seguro, mantendo uma observação atenta do bebê. Independentemente de como o bebê é amamentado, mantê-lo por perto deve significar que a mãe é capaz de responder a sugestões precoces de alimentação (inquietação, sons murmurantes, chupar o dedo) antes que o bebê acorde completamente e comece a chorar ${ }^{30}$.

No presente estudo, apenas $25 \%$ dos cuidadores trabalhavam fora (sem contar com o tempo de licença maternidade). Estudo prévio verificou que a ocupação materna "dona de casa" está relacionada a piores escores socioemocionais, ou seja, a mãe empregada apresenta maior segurança financeira e consequente bem-estar, transmitindo isso ao bebê $\hat{e}^{12}$. Entretanto entendese que seja muito importante que se cumpra o período de licença maternidade em pelo menos seis meses.

Um dado interessante neste estudo é que, embora 75\% dos bebês morassem com seus pais, $60 \%$ das mães se consideravam solteiras. Pesquisa prévia mostra que a presença paterna é considerada um fator protetor no ambiente familiar, como também, uma variável de impacto 
persistente no desenvolvimento do bebê, influenciando o cuidado, a organização e as oportunidades motoras disponibilizadas, isto é, os bebês que possuem essa condição apresentam inclusive melhor desenvolvimento de linguagem ${ }^{6}$.

Algumas limitações deste estudo devem ser consideradas. A amostra pequena, de 20 bebês avaliados, apenas no primeiro trimestre de vida, não permite que a análise estatística seja mais robusta, e que os resultados sejam excedidos para a totalidade da população. Além disso, a avaliação da motricidade fina deveria ter sido realizada, para que a população pudesse apresentar a pontuação composta e percentílica no desenvolvimento motor completo da Bayley III, como também não ter avaliado o domínio linguagem, cognitivo e comportamento adaptativo.

\section{Conclusão}

A literatura relata que bebês expostos em condições vulneráveis acabam apresentando desenvolvimento motor grosso e aspectos socioemocionais com atrasos bem importantes. $\mathrm{O}$ estudo, observou-se que isso não afetou o desenvolvimento motor bruto grosso associado com as habilidades socioemocionais no primeiro trimestre de vida dos bebês. Além disso, foi possível verificar uma associação positiva estatisticamente significativa entre idade gestacional, idade do bebê e peso ao nascer, com o escore motor grosso. Pode-se dizer, portanto, que quanto maior a idade gestacional, a idade do bebê e o peso ao nascer, maior foi o escore no desenvolvimento motor grosso.

Fica um convite aos fisioterapeutas para que haja pesquisas focadas num acompanhamento longitudinal a esse público, para que, assim, possam aprofundar se haverá mudanças no desenvolvimento motor grosso e socioemocional, já que os bebês estarão adquirindo novos marcos no desenvolvimento global. Assim como, ofertar aos pais oportunidades de conhecimento sobre o desenvolvimento dos seus filhos.

Outro fator a ser pensado diz respeito a estratégias de políticas públicas voltadas ao desenvolvimento de bebês pertencentes a ambientes vulneráveis, pois, nos primeiros anos de vida, o desenvolvimento global está em sua maior plenitude.

\section{Referências}

1. Madashi V, Mecca TP, Macedo EC, Paula CS. Bayley-III scales of infant and toddler development: transcultural adaptation and psychometric properties. Paidéia. 2016;26(64):189197. 
2. Fernandes PV, Gerzson LR, Almeida CS, Spessato BC. Desenvolvimento da manipulação do bebê em diferentes idades motoras. R. bras. Ci. e Mov. 2017;25(1):99-108.

3. Danielli CR, Farias BL, Santos DAPB, Neves FE, Tonetta MC, Gerzson LR et al. Efeitos de um programa de intervenção motora precoce no desenvolvimento de bebês em um abrigo residencial. ConScientiae Saúde. 2016;15(3):370-377.

4. Araujo LB, Mélo TR, Israel VL. Low birth weight, family income and paternal absence as risk factors in neuropsychomotor development. J Hum Growth Dev. 2017;27(3): 272-280.

5. Çelikkiran S, Bozkurt H, Coşkun M. Denver Developmental Test Findings and their relationship with sociodemographic variables in a large community sample of 0-4-year-old children. Noro Psikiyatr Ars. 2015;52(2):180-184.

6. Zago JTC, Pinto PAF, Leite HR, Santos JN, Morais RLS. Associação entre o desenvolvimento neuropsicomotor e fatores de risco biológico e ambientais em crianças na primeira infância. Rev CEFAC. 2017;19(3):320-329.

7. Pufal EC, Müller AB, Bandeira PFR, Valentini NC. Motor development in the hospitalized infant and its biological and environmental characteristics. Clin Biomed Re. 2018;38(1):6673.

8. Pereira KR, Valentini NC, Saccani R. Brazilian infants' motor and cognitive development: longitudinal influence of risk factors. Pediatr Int. 2016;58(12):1297-1306.

9. Gerzson LR, Azevedo KA, Demarco PR, Catarino BM, Palma MS, Almeida CS. O berçário da escola pública: aplicação de um programa de intervenção motora oportuna. Rev Fisioter Bras. 2017;18(4):417-25.

10. Hadders-Algra M. Early human motor development: from variation to the ability to vary and adapt. Neurosci Biobehav Rev. 2018;90:411-427.

11. Brasil. Ministério da Saúde. Diretrizes de estimulação precoce crianças de zero a 3 anos com atraso no desenvolvimento neuropsicomotor. Brasília: Ministério da Saúde; 2016. [citado em 01 nov 2019]. Disponível em:

<http://bvsms.saude.gov.br/bvs/publicacoes/diretrizes_estimulacao_criancas_0a3anos_neurop sicomotor.pdf $>$.

12. Metwally AM, Salah El-Din EM, Shehata MA, Shaalan A, El Etreby LA, Kandeel WA et al. Early life predictors of socio-emotional development in a sample of egyptian infants. PLoS One. 2016;11(7):e0158086.

13. Pereira KRG, Saccani R, Valentini NC. Cognição e ambiente são preditores do desenvolvimento motor de bebês ao longo do tempo. Fisioter. Pesqui. 2016;23(1):59-67.

14. Silva DI, Verissimo MLOR, Mazza VA. Vulnerabilidade no desenvolvimento infantil: influência das políticas públicas e programas de saúde. Rev bras cresc desen hum.

2015;25(1):11-18. 
15. Müller AB, Saccani R, Valentini NC. Impact of compensatory intervention in 6- to 18month-old babies at risk of motor development delays. Early Child Dev Care.

2017;187(11):1707-1717.

16. Neves K da R, Morais RL de S, Teixeira RA, Pinto PA. Growth and development and their environmental and biological determinants. J Pediatr. 2016;92(3):241-50.

17. Borba LS, Pereira KRG, Valentini NC. Motor and cognitive development predictors of infants of adolescents and adults mothers. J. Phys. Educ. 2017; 28(1):e-2811.

18. Bayley N. Bayley scales of infants and toddler development. 3th ed. São Paulo: Pearson Clinical Brasil; 2018.

19. Gesell AL. A criança dos 0 aos 5 anos. São Paulo: Martins Fontes; 2003.

20. Dusing SC. Postural variability and sensorimotor development in infancy. Dev Med Child Neurol. 2016;58 Suppl 4:17-21.

21. Adolph KE, Franchak JM. The development of motor behavior. Wiley Interdiscip Rev Cogn Sci. 2017;8(1-2).

22. Einspieler C, Peharz R, Marschik PB. Fidgety movements - tiny in appearance, but huge in impact. J Pediatr. 2016;92(3 Suppl 1):S64-70.

23. Mendonça B, Sargent B, Fetters L. Cross-cultural validity of standardized motor development screening and assessment tools: a systematic review. Dev Med Child Neurol. 2016;58(12):1213-1222.

24. Ribeiro CC, Pachelli MRO, Amrala NCO, Lamonica DAC. Habilidade do desenvolvimento de crianças prematuras de baixo peso e muito baixo peso. CoDAS. 2017;29(1):e20160058.

25. Formiga CKMR, Tudella E, Marques LR, Fagundes RR, Amaral LEF do, Linhares MBM. Desenvolvimento motor de bebês pré-termo e a termo de 0 a 6 meses de idade. Pediatria Moderna. 2015;(12): 422-426.

26. Silva TR, Rossetto EG, Souza SNDH, Baena JÁ. A incidência de reinternações entre prematuros de muito baixo peso e suas associações. Rev Varia Scientia - Ciências da Saúde. 2015;1(2):119-129.

27. Silva CP. O método em Marx: a determinação ontológica da realidade social. Serv Soc Soc. 2019;134:34-51.

28. Doulabi MA, Sajedi F, Vameghi R, Mazaheri MA, Baghban AA. Socioeconomic status index to interpret inequalities in child development. Iran J Child Neurol. 2017;11(2):13-25.

29. Furtado MAS, Mendonça ASGB, Lameira ABC, Ferreira LF. Avaliação do desenvolvimento motor de lactentes dependentes de servidores em instituição de ensino superior no Amazonas. Saúde e desen hum. 2018;6(1):29-38. 
30. UNICEF. United Kingdom. The baby friendly initiative. Caring for your baby at night. 2017. [citado em 06 nov 2019]. Disponível em:

$<$ https://www.unicef.org.uk/babyfriendly/baby-friendly-resources/sleep-and-night-timeresources/caring-for-your-baby-at-night/>. 